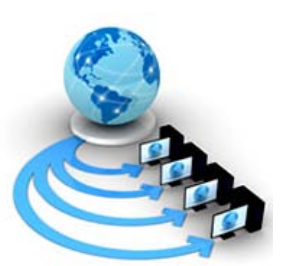

Volume 8, No. 8, September-October 2017

International Journal of Advanced Research in Computer Science

REVIEW ARTICLE

Available Online at www.ijarcs.info

\title{
DIGITAL IMAGE WATERMARKING TECHNIQUES: A REVIEW
}

\author{
Amarjot Kaur \\ M.TECH Scholar \\ Amritsar College of Engineering \& Technology \\ Amritsar (Punjab), India \\ Jagdeep Singh \\ Associate Professor \\ Amritsar College of Engineering \& Technology \\ Amritsar (Punjab), India
}

\begin{abstract}
This paper represents different Digital Watermarking Techniques which permits an individual to add hidden copyright notice as well as any other verification messages to images so that it can be protected from the unauthorized access. Generally watermark is provided with two types one is visible watermark and other is invisible watermark. The main aim of paper is to explore the comparison of various digital image watermarking techniques and it also demonstrates that all these watermarking techniques provides image watermarking with full security and reasonable capacity.
\end{abstract}

Keywords: Digital watermarking, Discrete Wavelet Transform, Singular value decomposition, Artificial Bee Colony.

\section{INTRODUCTION}

Watermarking is a process to full cover up some data that will be called watermark or label into the unique data (image, music or video) in ways that the watermark could either eliminated or acknowledged later to create an assertion in relation to the main object [1]. Image watermarking is an effective method to learn the unofficial use of authorized images. This method positions key data into sponsor picture which assists to locate the rightful control of image [2]. Generally digital image watermarking is just a way which is used to embed key data with some extra information in the original image which could be found when it will be required for different applications such as validation, operator recognition, material safety and trademark safety etc [3]. Often the scaling factor can also be employed for watermark embedding [4]. Fig 1 is given below which shows an apparent watermark and hidden watermark employed into host images.

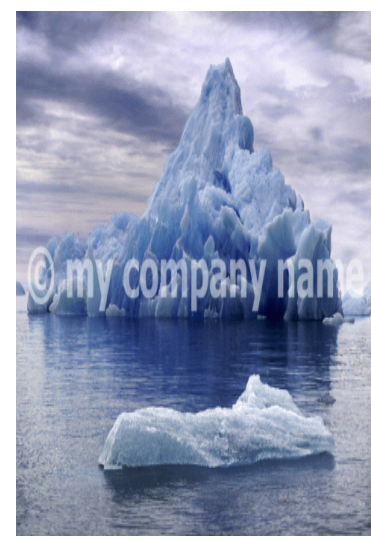

(a) Apparent Watermark

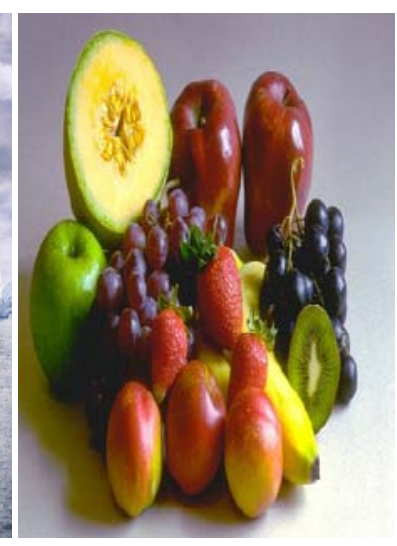

(b) Hidden Watermark
A. Watermarking System Fig 1

In Watermarking scheme, watermark is employed into original image, for the purpose of authenticating the host [5]. Fig 2 reveals the step by step process of embedding watermark into the original image. Later on, because of noise or other attacks degraded image is obtained which further detects the watermark which was earlier embedded into the host image.

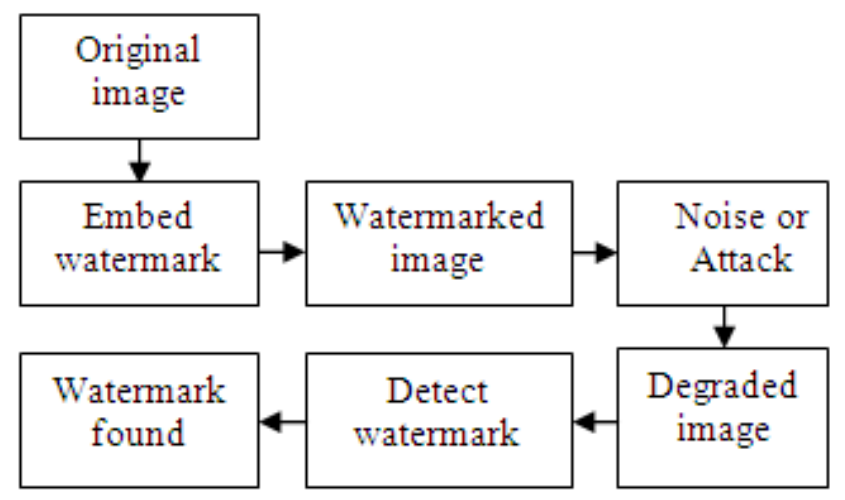

Fig 2: Typical watermarking block diagram

\section{B. Top features of Digital Watermarking}

1) Robustness: Watermark is said to be robust if it remains same after some attack [6]. An embedded watermark is robust if it is not altered after a variety of operations and manipulations for instance scaling, filtering, compression etc.

2) Imperceptibility: If quality of first picture can never get influenced after embedding watermark then it is said to be imperceptible. Hence watermark should be invisible to human eyes [7].

3) Security: Watermark is said to be secure only if an attacker is unable to eliminate the watermark without the knowledge of algorithm which was used for embedding [8]. Unauthorized parties can never access the watermark.

4) Capacity: Capacity or volume defines the number of bits or the maximum information which can be embedded into the host image [9].

5) Computational cost: Computational costs will be high if the complex algorithms are used for watermarking because complex algorithms always use more hardware as well as software resources. To reduce the computational costs, watermarking methods should be less complex [10]. 


\section{WATERMARKING TECHNIQUES}

\section{A. Discrete Wavelet Transform}

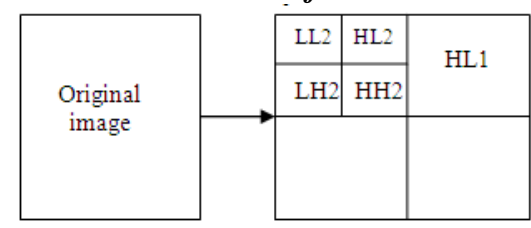

Fig 3: Workflow of 2 Level DWT

The image split into four sub-bands by DWT: minimum frequency sub-band (LLe) and other different subbands i.e. horizontal large frequency detail sub-band (HLe), vertical large frequency detail sub-band (LHe), diagonal large frequency detail sub-band (HHe), wherever ' $\mathrm{e}$ ' indicates the level of wavelet decomposition [11]. To be able to choose probably the most suitable sub-band to place the watermark, the secured watermark is stuck into four various sub-bands with various embedding intensities, respectively. Efficiency of picture watermarking applying DWT could be increased by raising the levels of DWT i.e. 2-level DWT, 3-level DWT and so on [12]. The watermark is stuck in the lower area of the cover picture and occurred watermarked picture is better quality without dropping the grade of the image. Other than this the large frequency sub-bands are not chosen for watermarking. Fig 3 shows the workflow of 2 Level DWT.

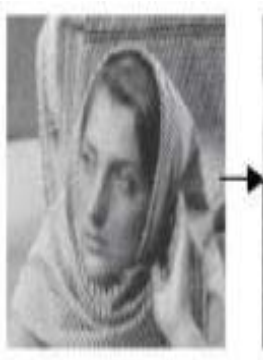

(a)

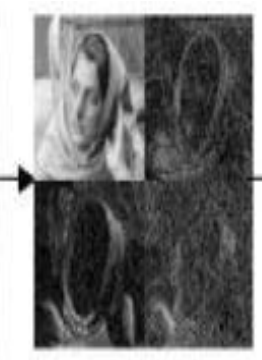

(b)

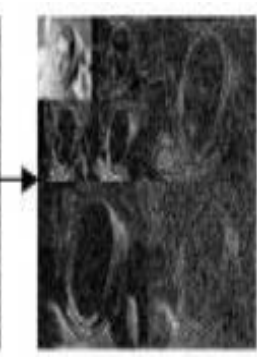

(c)
Fig: 4

As shown in fig 4, an image is divided into various different sub bands. The LL sub band is further decomposed into four other sub-bands. The LL sub band holds maximum information.

\section{B. Singular Value Decomposition}

By perception of picture processing, a picture might be considered as the matrix with nonnegative scalar entries. SVD is really an emphatic mathematical evaluation tool applied to analyze matrices. The SVD of a picture $\mathrm{M}$ with size $\mathrm{X}^{\times} \mathrm{x}$ is written by $\mathrm{M}=\mathrm{USV}^{\mathrm{T}}$, wherever $\mathrm{U}$ and $\mathrm{V}$ both are orthogonal matrices, and $S=\operatorname{diag}(\lambda \mathrm{i})$ is just a diagonal matrix of singular values $\lambda \mathrm{i}, \mathrm{i}=1, \ldots, \mathrm{m}$, that should be organized in descending order[13]. Columns of $U$ and $V$ would be the left singular vectors and right singular vectors respectively of picture $M$. These watermarking methods are mainly used for obtaining either SVD of original picture or each block of host picture, furthermore it alters singular values to the watermark [14]. The principal component matrix is given as following.

$\mathrm{PC}=\mathrm{U} \times \mathrm{S}$

\section{Arnold Transform}

Image scrambling determines change of the image which rearranges the spatial place of the pixels according to the rules, and makes image distortion for the purpose of security [15]. If the change rules and guidelines weren't provided, the initial image can't be reconstructed. Regular techniques for scrambling contain Arnold change, Miraculous change, Fractal Hilbert bend, Conway sport and Gray code change etc [16]. Arnold transform is surely an effective as well as strong iterative technique. It is used to provide randomization to the elements of any array and it could only be solved right back with the help of unique key. That stops the unauthorized accessibility of watermark despite with the successful extraction [17]. A matrix $P \times Q$ of an image having height $\mathrm{N}$ is scrambled by method:

$\left(\begin{array}{l}\mathrm{P}^{\prime} \\ \mathrm{Q}^{\prime}\end{array}\right)=\left(\begin{array}{ll}1 & 1 \\ 1 & 2\end{array}\right)\left(\begin{array}{l}\mathrm{P} \\ \mathrm{Q}\end{array}\right) \bmod (\mathrm{N})$

Where $(\mathrm{P}, \mathrm{Q})$ is location of original image pixels, while (P',Q') are coordinates of transformed picture pixels [18].

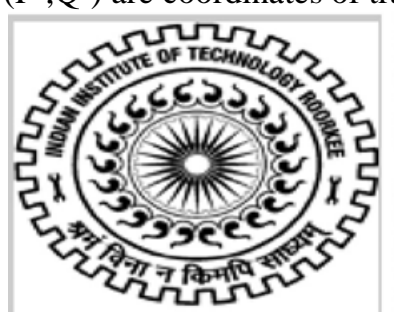

(a)

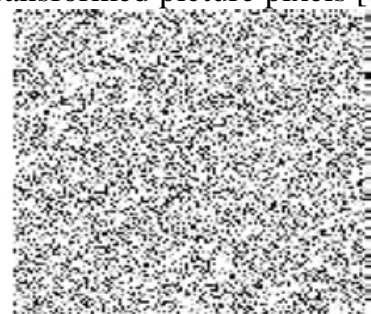

(b)
Fig: 5

Fig 5(a) gives a watermarked image and fig (b) gives an Arnold transformed watermarked image.

\section{Artificial Bee Colony}

$\mathrm{ABC}$ is really an easy and effective population based optimization algorithm. It is related to the intelligent foraging activities of the honey bee swarms. The probable alternatives are displayed by the food source in ABC and fitness of some of these alternatives is displayed by the nectar quantity of the food source [19]. In this algorithm, you will find three categories of bees in colony of artificial bee: employed bees, onlookers and scouts. The total quantity of employed bees is corresponding to how many foodstuff options are there across the hive. Employed bee whose food resource got forgotten by the bees becomes scout [20]. ABC contains three necessary components.

1) Food resources: Profitability of food resources is related with many facets for instance abundance of food, their closeness to nest and the easily getting the power from the source.

2) Employed bees: These bees are linked with particular food option which they exploited as well as hold information regarding the precise source [21]. They reveal these details with the forager bees that wait in the hive by dance that is a typical example of numerous interaction.

3) Unemployed bees: Unemployed bees contain onlookers and scouts. The scouts arbitrarily research food sources [22]. 


\begin{tabular}{|c|c|}
\hline 1) & $\begin{array}{l}\text { Algorithm: Artificial Bee Colony } \\
\text { Start }\end{array}$ \\
\hline 2) & $\begin{array}{l}\text { Start the solution population , } \\
\mathrm{j}=1, \ldots, \mathrm{SN}\end{array}$ \\
\hline 3) & Examine the population \\
\hline 4) & cycle $=1$ \\
\hline 5) & Repeat \\
\hline 6) & $\begin{array}{l}\text { Create new option } \mathrm{V}_{\mathrm{mj}} \text { for the } \\
\text { employed bees applying following } \\
\text { equation and consider those solutions. } \\
\mathrm{V}_{\mathrm{mj}}=\mathrm{x}_{\mathrm{mj}}+\varnothing_{\mathrm{mj}}\left(\mathrm{x}_{\mathrm{mj}}-\mathrm{x}_{\mathrm{kj}}\right)\end{array}$ \\
\hline 7) & $\begin{array}{l}\text { Keep the solution which is best among } \\
\text { recent and candidate }\end{array}$ \\
\hline 8) & $\begin{array}{l}\text { Choose the visited option for onlooker } \\
\text { bees according with their fitness }\end{array}$ \\
\hline 9) & $\begin{array}{l}\text { Create new option } v_{m j} \text { for Onlooker } \\
\text { bees applying following equation and } \\
\text { evaluate them }\end{array}$ \\
\hline & $\mathrm{V}_{\mathrm{mj}}=\mathrm{x}_{\mathrm{mj}}+\varnothing_{\mathrm{mj}}\left(\mathrm{x}_{\mathrm{mj}}-\mathrm{x}_{\mathrm{kj}}\right)$ \\
\hline 10) & $\begin{array}{l}\text { Keep the solution which is best among } \\
\text { recent and candidate }\end{array}$ \\
\hline 11) & $\begin{array}{l}\text { If an abandoned food resource occurs } \\
\text { then change it by employing a scout } \\
\text { bee }\end{array}$ \\
\hline 12) & $\begin{array}{l}\text { Save into storage the very best option } \\
\text { which is obtained up to now }\end{array}$ \\
\hline 13) & cycle $=$ cycle +1 \\
\hline 14) & Till cycle $=$ MCN \\
\hline
\end{tabular}

In above algorithm of Artificial Bee Colony, first of all it initializes the solution population and evaluates it. Further it generates new solutions for employed bees and evaluates those generated solutions for keeping the very best option among current and the candidate. According to fitness of onlooker bees a visited solution is selected and later it generates new solutions for onlooker bees and evaluates those generated solutions. Now again keep the very best solution among current and candidate. Furthermore it checks for abandoned food source and if it exists then replace it with scout bee and it saves the best solution in memory.

\section{RELATED WORK}

Jagdish Chandra Patra et.al (2006) [3] had written about a better SVD-based approach which is more tolerant to various kinds of attacks. It increases the tenderness and consistency of the watermarking system.

Mingwei Zhao et.al (2008) [5] had presented a technique to eliminate connection among DWT coefficients by mixing the visible features of minimal frequency sub-image of DWT and capability of DCT. It will increase blind DCT algorithm for obtaining a new color picture digital watermarking scheme predicated on DWT and DCT.

Harikrishna Narasimhan (2009) [6] had shown a simultaneous edition of the algorithm for distributed storage architectures. All the three kinds of bees are split equally on the list of accessible processors. A couple of alternatives should be presented in the local storage of every processor. A replicate of every alternative can also be preserved in a worldwide distributed memory. Throughout each period, the group of bees at the processor improves the solutions in the local memory. By end of the period, alternatives are replicated into equivalent slots in the distributed storage and make it accessible to all or any other bees.

Chih-Chin Lai et.al (2010) [8] had planned a hybrid watermarking method which mixes the discrete cosine transformation and SVD for multimedia trademark safety and content authentication.

Siva Venkateswara Rao .V et.al (2012) [11] had planned a non-blind watermarking system for pictures predicated on SVD and PSO in DWT domain. Singular values of sponsor picture with principal components in the DWT domain are altered in order to provide trademark safety in addition to reliability. PSO is integrated for locating perfect scaling factors required for effective robustness and also to provide better security.

Prabhat Kumar Sharma et.al (2012)[12] had planned the utilization of $\mathrm{ABC}$ for optimum fusion of multi-temporal pictures and studied the effect of the difference in the source area.

Shilpa P.Metkar et.al (2013) [14] had presented a scheme for enhancing picture protection. Two techniques RC4 and Advances Encryption Standard are followed for security that employs stream cipher and block cipher respectively.

Jianting Guo et.al (2015) [15] planned an effective watermarking system in secured domain, which safeguards initial pictures from alternative party embedders. Watermark removal could be conducted on both the plaintext and the protected domains. The watermarking technique will offer ownership and copyright protection.

Sudhanshu Suhas Gonge et.al (2015) [19] planned an effective and protected DWT-SVD secured watermark for trademark security of Cheque image. Watermarking Strategy has employed in order to offer the ownership and security for cheque picture.

Irshad Ahmad Ansari et.al (2016) [21] planned a guaranteed picture watermarking having good capacity DWT applied on sponsor picture and later block wise single components should employ in order to insert a principal component.

\section{COMPARISON TABLE}

\begin{tabular}{|c|c|c|c|}
\hline S.NO. & Technique & Feature & Limitation \\
\hline 1 & DWT-DCT & $\begin{array}{c}\text { Increased Robustness, Increased } \\
\text { Security }\end{array}$ & $\begin{array}{r}\text { Size of watermark which should } \\
\text { embedded is restricted }\end{array}$ \\
\hline
\end{tabular}


Amarjot Kaur et al, International Journal of Advanced Research in Computer Science, 8 (8), Sept-Oct 2017,714-718

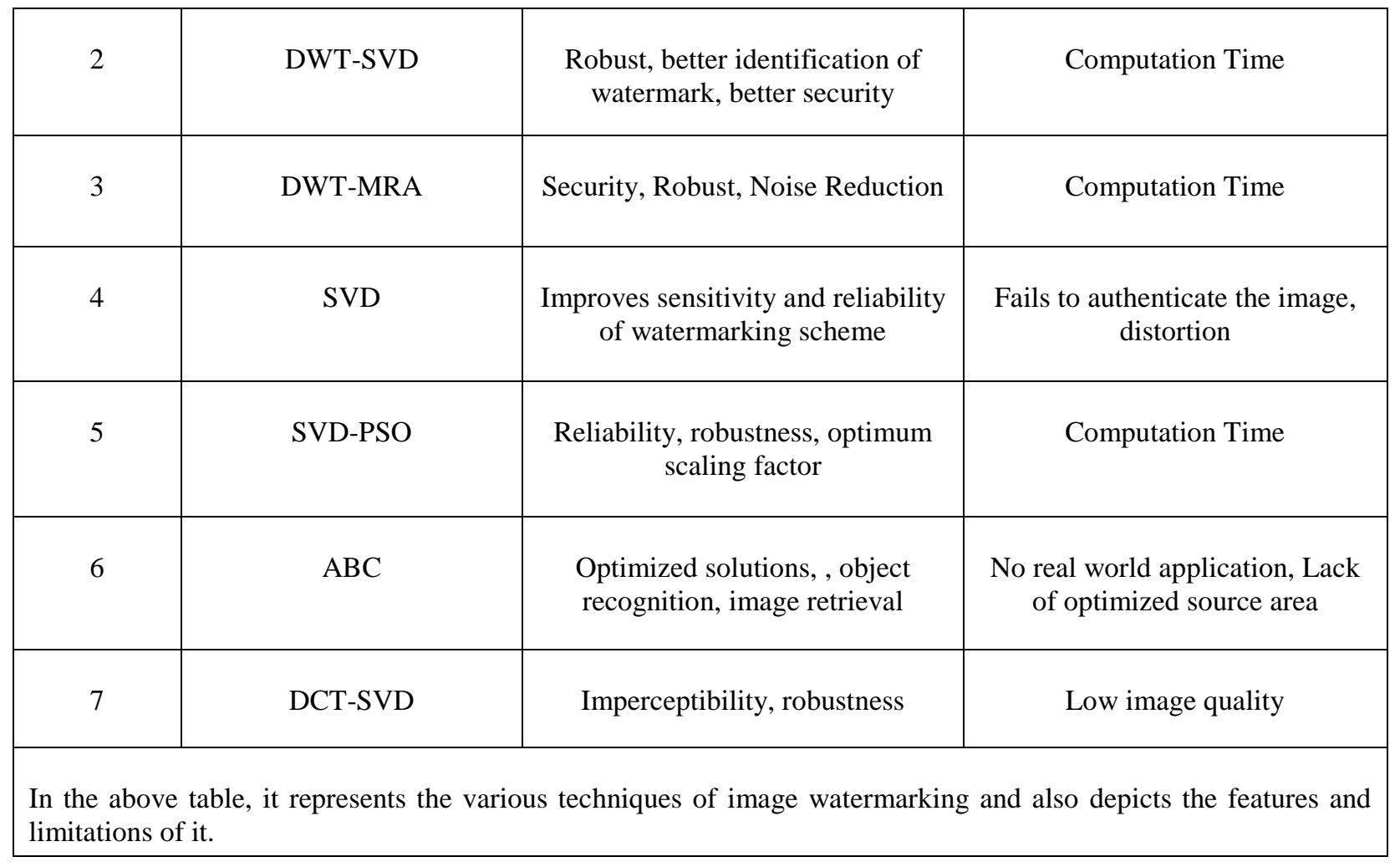

\section{GAPS IN LITERATURE}

Many research gaps which are determined using the literature review are explained as follows:

1. The effect of the watermark scrambling on a provided watermark picture has been neglected by many of the existing researchers.

2. The change of the SVD method is neglected by many existing researchers.

3. The usage of principal component has been ignored by existing researchers.

\section{CONCLUSION}

A digital watermarking is probably the strong implies to uncover the particular unauthorized uses of copyrighted image. So this paper shows about the comparison of various techniques based on image watermarking. This paper indicates that the $\mathrm{ABC}$ for watermarking has improved results when implemented on the DWT, SVD techniques but there are some issues such as the use of watermark scrambling still are unaddressed. In near future a new technique will be proposed which will improve the speed and security of the watermarking technique further.

\section{REFERENCES}

[1] Gary L. Friedman, The trustworthy digital camera: restoring credibility to the photographic image, IEEE Trans. Consum. Electron. 39 (4) (1993)905-910.

[2] V.M. Potdar, S. Han, E. Chang, A survey of digital image watermarking techniques, 3rd IEEE International Conference on Industrial InformaticsINDIN'05 (2005) 709-716.

[3] Patra, Jagdish Chandra, et al. "An improved SVD-based watermarking technique for image and document authentication." Circuits and Systems, 2006. APCCAS 2006. IEEE Asia Pacific Conference on. IEEE, 2006.
[4] Karaboga, Dervis, and Bahriye Basturk. "Artificial bee colony (ABC) optimization algorithm for solving constrained optimization problems." International Fuzzy Systems Association World Congress. Springer Berlin Heidelberg, 2007.

[5] Zhao, Mingwei, and Yanzhong Dang. "Color image copyright protection digital watermarking algorithm based on DWT \& DCT." Wireless Communications, Networking and Mobile Computing, 2008. WiCOM'08. 4th International Conference on. IEEE, 2008.

[6] Narasimhan, Harikrishna. "Parallel artificial bee colony (PABC) algorithm." Nature \& Biologically Inspired Computing, 2009. NaBIC 2009. World Congress on. IEEE, 2009.

[7] Gaurav Bhatnagar, Balasubramanian Raman, A new robust reference watermarking scheme based on DWT-SVD, Comput. Stand. Interfaces 31 (5)(2009) 1002-1013.

[8] Lai, Chih-Chin, and Chih-Hsiang Yeh. "A hybrid image watermarking scheme based on SVD and DCT." Machine Learning and Cybernetics (ICMLC), 2010 International Conference on. Vol. 6. IEEE, 2010.

[9] Chih-Chin Lai, Cheng-Chih Tsai, Digital image watermarking using discrete wavelet transform and singular value decomposition, IEEE Trans.Instrum. Meas. 59 (11) (2010) 3060-3063.

[10] Lin Weisi, Dacheng Tao, Janusz Kacprzyk, Zhu Li, Ebroul Izquierdo, Haohong Wang, Multimedia Analysis, Processing and Communications, vol. 346,Springer, 2011.

[11] Rao, V. Siva Venkateswara, Rajendra S. Shekhawat, and V. K. Srivastava. "A reliable digital image watermarking scheme based on SVD and particle swarm optimization." Engineering and Systems (SCES), 2012 Students Conference on. IEEE, 2012.

[12] Sharma, Prabhat Kumar, et al. "Artificial bee colony and its application for image fusion." International Journal of Information Technology and Computer Science (IJITCS) 4.11 (2012): 42.

[13] Chen, Yongchang, Weiyu Yu, and Jiuchao Feng. "A reliable svd-dwt based watermarking scheme with artificial bee colony algorithm." International Journal of Digital Content Technology and its Applications 6.22 (2012): 430. 
[14] Metkar, Shilpa P., and Milind V. Lichade. "Digital image security improvement by integrating watermarking and encryption technique." Signal Processing, Computing and Control (ISPCC), 2013 IEEE International Conference on. IEEE, 2013.

[15] Zheng, Peijia, and Jiwu Huang. "Discrete wavelet transform and data expansion reduction in homomorphic encrypted domain." IEEE Transactions on Image Processing 22.6 (2013): 2455-2468

[16] Musrat Ali, Chang Wook Ahn, Millie Pant, A robust image watermarking technique using SVD and differential evolution in DCT domain, Opt.: Int. J.Light Electron Opt. 125 (1) (2014) 428-434.

[17] Nasrin M. Makbol, Bee Ee Khoo, A new robust and secure digital image watermarking scheme based on the integer wavelet transform and singular value decomposition, Digital Signal Process. 33 (2014) 134-147.

[18] Guo, Jianting, Peijia Zheng, and Jiwu Huang. "Secure watermarking scheme against watermark attacks in the encrypted domain." Journal of
Visual Communication and Image Representation 30 (2015): 125-135.

[19] Gonge, Sudhanshu Suhas, and Ashok Ghatol. "A Robust and Secure DWT-SVD Digital Image Watermarking Using Encrypted Watermark for Copyright Protection of Cheque Image." International Symposium on Security in Computing and Communication. Springer International Publishing, 2015.

[20] Harjito, Bambang, and Heri Prasetyo. "False-positive-free GSVD-based image watermarking for copyright protection." Electronics and Smart Devices (ISESD), International Symposium on. IEEE, 2016.

[21] Ansari, Irshad Ahmad, Millie Pant, and Chang Wook Ahn. "ABC optimized secured image watermarking scheme to find out the rightful ownership." Optik-International Journal for Light and Electron Optics 127.14 (2016): 5711-5721.

[22] Choudhary, Rita, and Girish Parmar. "A robust image watermarking technique using 2-level discrete wavelet transform (DWT)." Communication Control and Intelligent Systems (CCIS), 2016 2nd International Conference on. IEEE, 2016. 